\title{
Die Funktionen anaphorischer Proformen beim Simultandolmetschen aus dem Deutschen1
}

\begin{abstract}
When working from German in the simultaneous mode, interpreters often start producing the target language utterance before they have heard the end of the source language utterance in question. The reason for this is that the short-term memory may become overloaded if the interpreter decides to wait for the whole utterance to be complete. As soon as he has captured the full meaning of the German utterance (or a meaning unit of it) he will take the incomplete output segment up again by means of anaphoric pro-forms (such as resumptive pronouns), correct it, if necessary, and/or complete it in accordance with the requirements of his target language production. This paper shows that, as they may be used for various functions in the process, anaphoric pro-forms are a flexible processing device.
\end{abstract}

\section{Einleitung}

Ausgangspunkt der folgenden Überlegungen ist das bekannte Phänomen, das beim Simultandolmetschen (SD) aus dem Deutschen notorisch Schwierigkeiten bereitet, nämlich das topologische Grundprinzip der deutschen Sprache, das als Satzklammer (oder genauer: Rahmenbildung) bezeichnet wird. Damit ist die "Distanzstellung der Prädikatsteile und anderer (syntaktisch eng zusammengehöriger) Elemente" gemeint (Bußmann 1990:662), eine Besonderheit der deutschen Ausgangssprache (AS), die beim Hörer eine u.U. sehr späte Disambiguierung des Sinnzusammenhangs bewirkt. Dadurch werden beim SD syntaktische Umstrukturierungen erforderlich, die die begrenzte Verarbeitungskapazität des Dolmetschers belasten. Insbesondere werden erhöhte Anforderungen an die Gedächtniskapazität gestellt, was die verfügbaren Ressourcen für die beiden anderen Verarbeitungsoperationen (Hören und Verstehen des laufenden Inputs, zielsprachliche Produktion) einschränkt, es sei denn, der Dolmetscher benutzt effiziente Produktionsstrategien.

Bewußter Umgang mit den begrenzten Verarbeitungsressourcen bewirkt, daß der Dolmetscher in den meisten Fällen mit der zielsprachli-

1 Der nachstehende Artikel ist die überarbeitete Version einer Manuskriptfassung, die ich im Rahmen des "Translation Studies Congress" 1992 in Wien vorgetragen habe. 
chen (ZS-) Produktion beginnt, auch wenn ihm der Sinn der betreffenden ausgangssprachlichen Äußerungseinheit noch nicht klar geworden ist.

Bei dieser Ausgangslage wird sich der Dolmetscher in besonderem Maße um Kohäsion und Kohärenz seiner ZS-Produktion bemühen.

Er wird dies durch verstärkten Einsatz von kohäsiven Mitteln tun, die explizit, an der Textoberfläche Zusammenhang signalisieren.

Das prototypische Mittel für die Signalisierung des Zusammenhangs von Textteilen sind anaphorische Pronomen.

So ist es laut u.a. Halliday \& Hasan der anaphorischen Funktion des Pronomens zu verdanken, daß im Standardfall von zwei aufeinander folgenden Sätzen mit einem nominalen Antezedens im ersten Satz und pronominaler Wiederaufnahme im zweiten ein Text entsteht, daß die beiden Sätze als Einheit interpretiert werden (1976:2).

Hierauf basiert die erste Grundannahme:

Im Output des Simultandolmetschers bei der Übertragung aus dem Deutschen sind relativ viele Vorkommensfälle von Wiederaufnahme durch Pronomen und andere, funktionsähnliche Proformen zu erwarten, die kein Äquivalent in der entsprechenden AS-Äußerungseinheit haben. ${ }^{2}$

\subsection{Was sind Proformen, und was leisten sie insbesondere aus der Sicht des Textproduzenten?}

Proformen sind mit Beaugrande \& Dressler (1981) "ökonomische, kurze Wörter ohne besonderen Inhalt, die für determinierte, inhaltsaktivierende Ausdrücke an der Oberfläche des Textes einstehen können ...".3 Proformen "erlauben Textbenutzern, den Inhalt im aktiven Gedächtnisspeicher handhabbar zu halten, ohne alles wiederholen zu müssen" (a.a.O.: 64).

2 Anaphorische Proformen in der ZS-Produktion, die durch ein AS-Äquivalent bedingt sind, geben wenig Aufschluß über Verarbeitungsstrategien des Dolmetschers.

3 Da auch mehrsilbige Wörter und mehrteilige Syntagmen vom Typ "diese Dinge" als Proformen verwendet werden, bedarf die Formulierung "kurze Wörter" einer Präzisierung bzw. Relativierung. Mit der Charakterisierung, daß eine Proform "kürzer ist als der Ausdruck, den sie ersetzt", sorgen Beaugrande \& Dressler selbst an anderer Stelle (a.a.O.:69) für die nötige Relativierung. Trotz solcher offensichtlichen Mängel ist jedoch Beaugrande \& Dresslers Proform-Definition wegen des kognitionstheoretisch fundierten prozeduralen Ansatzes einer korrekten, aber ausschließlich syntaktisch orientierten Definition (wie z.B. der von Bußmann) vorzuziehen. 
"Eine Pro-Form verkleinert die Verarbeitungsmühe, da sie kürzer ist als der Ausdruck, den sie ersetzt" (a.a.O.:69). Proformen sorgen m.a.W. für die "Ökonomie von Material und Verarbeitungsaufwand" (a.a.O.:51).

Wegen dieser Eigenschaften sehen Beaugrande \& Dressler die primäre Motivation für den Gebrauch von Proformen überhaupt im Kriterium der Effizienz (a.a.O.69), wobei Effizienz zur Verarbeitungsleichtigkeit beiträgt, d.h. "zur Durchführung von Operationen mit geringer Belastung der Potentiale ... von Aufmerksamkeit und Zugriff" (a.a.O.:35).

Dies ist der Hintergrund für die zweite Grundannahme:

Die Wiederaufnahme einer unmittelbar vorher erwähnten ZS-Äußerungseinheit bzw. eines Äußerungsteils durch Proformen kann als Angelpunkt effizienter Produktionsstrategien betrachtet werden.

\subsection{Welche Proformen werden besonders häufig auftreten?}

Proformen mit wenig spezifiziertem Referenzpotential sind besonders breit anwendbar und können z.T. höchst unterschiedliche Einheiten wiederaufnehmen (von Wörtern bis zu Sätzen), unabhängig vom syntaktischen Status dieser Einheiten. Zu Proformen mit diesen Eigenschaften gehören vor allem das Personal- und Demonstrativpronomen der 3. Person Singular Neutrum, aber auch andere Proformen mit entsprechender Stellvertreterfunktion, z.B. Zusammenfassungen vom Typ "diese Dinge"/"diese Verhältnisse" oder "alles dies". Daher ist anzunehmen, daß gerade Proformen mit großer Extension sehr frequent sind. Da Pronomen überhaupt die gängigsten Proformen sind, benutze ich das Kürzel ProW (= pronominale Wiederaufnahme) für die Verwendung breit anwendbarer anaphorischer Proform-Varianten generell.

\section{Die Funktionen von ProW im SD-Prozeß}

Zur Prüfung der angeführten Grundannahmen und zum Zwecke der Ermittlung und Beschreibung der Funktionen, die ProW im on-lineProzeß erfüllt, habe ich eine empirische Untersuchung zum Thema Anaphorische Proformen beim SD aus dem Deutschen durchgeführt. Als AS-Quelle der Untersuchung dienten Debattenbeiträge aus den Ausführlichen Sitzungsberichten des Europäischen Parlaments. Die Texte, die eine für die Zwecke der Untersuchung ausreichende Anzahl von Beispielen für verschiedene Klammertypen enthalten, wurden von mir 
selbst als "native speaker" vorgetragen und von 8 Versuchspersonen gedolmetscht (4 erfahrenen Berufsdolmetschern und 4 auszubildenden Dolmetschern). Aus diesem Material stammen die unten angeführten Beispiele.

Generell ist zu sagen, daß die verschiedenen Funktionen von ProW nicht immer eindeutig voneinander zu trennen sind, aber es lassen sich mehrere Hauptfunktionen unterscheiden, von denen ich nur einige wenige herausgreifen will.

\subsection{Korrektur von Prognosefehlern}

Konstruktionen mit ProW können zur Korrektur von Prognosefehlern genutzt werden, d.h. Fehlern, die auf Antizipation zurückzuführen sind. Antizipation ist, wenn sie gelingt, zweifellos die effizienteste Verarbeitungsstrategie. Wiederaufnahme zur nachträglichen Output-Optimierung ist dann nicht erforderlich. Aber wenn der Dolmetscher korrigieren muß, kann er das tun, indem er seinen Output im Vergleich zum Input expandiert, und zwar typisch durch eine syntaktisch selbständige Prädikation, einen Äußerungsteil b, in dem die anaphorische Proform Thema oder thematisches Element ist, während der korrigierte Informationsteil das Rhema bildet:

$\begin{array}{ll}\begin{array}{l}\text { Äußerungsteil a } \\ \text { mit Antizipation }\end{array} & +\quad \begin{array}{l}\text { Äußerungsteil b } \\ \text { mit Proform als Thema(-Element) und } \\ \text { Korrektur als Rhema }\end{array}\end{array}$

Beispiel (1):

AS:

Wir können den Verlust keines einzigen Menschenlebens dadurch entschuldigen, daß wir erklären, die Schuld trage der Diktator Saddam Hussein.

ZS:

Vi kan ikke acceptere at der mistes et eneste liv og vi kan ikke undskylde det med at det er Saddam Hussein, diktatoren, der er skyld i det.

(deutsch: Wir können nicht akzeptieren, daß ein einziges Leben verlorengeht, und wir können das nicht damit entschuldigen, daß es S.H., der Diktator ist, der Schuld daran ist.)

In diesem Beispiel wird das falsch antizipierte "akzeptieren" durch eine Korrekturstruktur mit ProW richtiggestellt. Dabei wird die wichtige Leistung von ProW augenfällig, nämlich das sprachökonomische Gegenwärtighalten eines eben erwähnten Inhalts, ohne daß der ganze Inhalt, hier der zu korrigierende Äußerungsteil a, wiederholt werden muß. 


\subsection{Nachtrag zu Output-Elementen}

Eine ähnliche Konstellation ergibt sich, wenn der Dolmetscher einen Äußerungsteil b hinzufügt, der einen Nachtrag zu Äußerungsteil a darstellt. Äußerungsteil a enthält typisch, aber nicht notwendigerweise, eine Antizipation.

$\begin{array}{lll}\begin{array}{l}\text { Äußerungsteil } \mathrm{a} \\ \text { +/- Antizipation }\end{array} & +\quad \begin{array}{l}\text { Äußerungsteil b } \\ \text { mit Proform als Thema(-Element) } \\ \text { u. Ergänzung/Präzisierung als Rhema }\end{array}\end{array}$

Der Expansionsteil wird entweder hinzugefügt, weil der Dolmetscher zunächst von der Verarbeitung eines AS-Informationselementes absieht, das dann im Äußerungsteil a fehlt, oder weil der Dolmetscher ein bestimmtes AS-Informationselement akustisch noch nicht rezipiert hat, während er dabei ist, die Formulierung von Äußerungsteil a abzuschlieBen.

Letzteres ist der Fall im folgenden Beispiel, wo die Unübersichtlichkeit des "daß"-Satzes (eine Kombination von Gliedsatzklammer und Nominalklammer) den Dolmetscher dazu veranlaßt, seinen Output durch eine nachträgliche Ergänzung zu vervollständigen:

Beispiel (2):

AS:

(Die Geiseln in Irak und Kuwait werden befreit.)

Wir freuen uns mit ihnen und ihren Familien und begrüßen die Tatsache, daß damit eine der in der Resolution des UNO-Sicherheitsrates gestellten Forderungen vom irakischen Präsidenten erfüllt wurde.

ZS:

Vi glæder os med dem og deres familier og vi hilser den kendsgerning velkommen at der dermed er opfyldt en af de betingelser der kom i resolutionen fra FNs Sikerhedsråd. Det er den irakiske præsident der nu har opfyldt denne betingelse.

(deutsch: (...) begrüßen die Tatsache, daß damit eine der Bedingungen erfüllt ist, die in der Resolution des UNO-Sicherheitsrates gekommen sind. Es ist der irakische Präsident, der nun diese Bedingung erfüllt hat.)

Der rhematische Charakter der Ergänzung spiegelt sich in der Satzspaltungsstruktur wieder ("Es ist der irakische Präsident, der..."), während der thematische Charakter der Proform u.a. durch die Rekurrenz des Substantivs aus Äußerungsteil a zum Ausdruck kommt. Die Sprachökonomie auch der etwas längeren Proform "denne betingelse" ("diese Bedingung") wird im Vergleich zu dem langen Nominalsyntagma deutlich, 
das durch sie wiederaufgenommen wird ("eine der Bedingungen, die in der Resolution des UNO-Sicherheitsrates gekommen sind").

\subsection{Zusammenfassung}

Zusammenfassende Wiederaufnahme eines unselbständigen Äußerungsteils a ist eine der wichtigsten ProW-Funktionen. Statt auf das sinnentscheidende Element zu warten, wird eine bereits rezipierte Teilinformation mit noch ungeklärtem syntaktischem und/oder semantischem Status in ZS produziert, so daß sie keine bzw. kaum Speicherressourcen mehr verbraucht. Sowie die gesamte AS-Äußerungseinheit rezipiert ist, kann der Dolmetscher mit Hilfe von ProW an die Teiläußerung anschließen und sie zu Ende führen.

Beispiel (3):

AS:

Nach Abschluß des Problems (...) müssen wir auch unsere Glaubwürdigkeit wiederherstellen, indem wir die anderen Probleme der Region, zu welchen genauso klare Resolutionen der UNO vorliegen, mit der gleichen Entschlossenheit angehen und ebenfalls zu einer friedlichen Lösung führen.

ZS:

Når problemet er blevet løst (...) må vi også genskabe vor egen troværdighed ... således at forstå at de andre problemer der er i regionen og hvor FN jo har forelagt lige så klare resolutioner, at disse problemer bliver taget op med samme beslutning / beslutsomhed og at dette også fører til en fredelig løsning.

(deutsch: Wenn das Problem gelöst ist, müssen wir auch unsere eigene Glaubwürdigkeit wiederherstellen in dem Sinne, daß die anderen Proble-

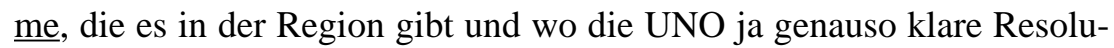
tionen vorgelegt hat, daß diese Probleme mit derselben Entschlossenheit aufgegriffen werden (...).)

Der Dolmetscher ändert hier die lineare Reihenfolge der Satzglieder in der AS-Äußerung. Statt mit dem kurzen AS-Subjekt "wir" beginnt er die ZS-Produktion des "indem"-Satzes (ein Beispiel für eine Gliedsatzklammer mit eingebettetem Gliedsatz zweiten Grades) mit dem langen ASAkkusativobjekt, das er auf diese Weise nicht zu speichern braucht. Dies geschieht im Rahmen einer "at"-Satz-Konstruktion (“daß"-Satz-K.), die nicht zu Ende geführt wird. An diese Teiläußerung schließt der Dolmetscher an, indem er die unvollendete "at"-Satz-Konstruktion wiederaufnimmt. Dabei wiederholt er nur die einleitende Konjunktion, der Rest 
wird durch die zusammenfassende Proform "disse problemer" ("diese Probleme") wiederaufgenommen, eine Lösung, die im Verhältnis zu dem langen, stark spezifizierten Bezugselement sprachökonomisch ist und darüberhinaus auch deswegen wenig Verarbeitungskosten bereitet, weil das rekurrente Element "problemer" bereits im Gedächtnis aktiviert ist. Die Wiederaufnahme im Rahmen der wiederholten "at"-Satz-Konstruktion in Beispiel (3) ist genau genommen "überflüssig", sie stimmt aber überein mit einem Charakteristikum der gesprochenen Sprache, wonach, besonders bei längeren Sequenzen, Konstruktionen, die durch Parenthesen oder abhängige Gefügeteile unterbrochen sind, wiederaufgenommen werden. ${ }^{4}$ Die eigentlich überflüssige zusammenfassende Wiederaufnahme kann als linguistischer Spiegel der mentalen Verarbeitungsprozesse betrachtet werden, die der Dolmetscher unter erschwerten Bedingungen vollzieht. Sie ist eine Art Rückversicherung für den Dolmetscher, der den Oberflächenwortlaut der ersten, von ihm selbst angefangenen Konstruktion vergessen haben kann, ihren Inhalt aber durch die anaphorische Proform gegenwärtig hält. Die zusammenfassende Wiederaufnahme hat zudem den Vorteil, beim Hörer das Verständnis zu sichern. ${ }^{5}$ Es ist auch als Vorzug zu betrachten, daß ProW mit einem weiteren Charakteristikum der gesprochenen Sprache übereinstimmt, nämlich der Tendenz, lange Vorfelder zu vermeiden. ${ }^{6}$ Die "überflüssige" Wiederaufnahme mit Hilfe einer kurzen Proform sorgt im zweiten Anlauf dafür, daß sich der Dolmetscher mehr im Einklang mit Konstruktionsweisen der gesprochenen Sprache ausdrücken kann.

\subsection{Verzögerungsstrategie}

Konstruktionen mit ProW kann der Dolmetscher auch als Verzögerungsstrategie anwenden, um Zeit zu gewinnen.

Beispiel (4):

AS: siehe Beispiel (3).

ZS:

Efter at problemet er løst (...) må vi også genskabe vor egen troværdighed. Det må vi gøre på den måde at de andre problemer i området som der også er kommet tydelige resolutioner fra FN om, at vi forsøger at

4 Dieses Phänomen ist als "Drehsatz" bekannt. Vgl. Heinze (1979).

5 Vgl. Bastian (1991).

6 Diese Tendenz stellen Galberg Jacobsen/Skyum Nielsen (1988) fürs Dänische fest. 
løse dem med samme beslutsomhed og forsøger at løse dem på en fredelig måde.

(deutsch: Nachdem das Problem gelöst ist, müssen wir auch unsere eigene Glaubwürdigkeit wiederherstellen. Das müssen wir auf die Weise tun. $\underline{\text { daß }}$ die anderen Probleme in dem Gebiet, zu denen auch deutliche Resolutionen der UNO gekommen sind, daß wir versuchen, die mit derselben Entschlossenheit zu lösen (...).)

Während der Dolmetscher einen Expansionsteil mit ProW produziert hier "Det må vi gøre på den måde at" ("Das müssen wir auf die Weise tun, daß”) - verfolgt er den laufenden Input und kann dann die Äußerung auf der Basis vollständiger Disambiguierung oder wesentlich sichererer Erwartung weiterführen. Der Expansionsteil ist im obigen Beispiel eine typische doppelte Wiederaufnahme-Konstruktion mit der Pro-Verb-Form "gøre" ("tun") und dem anaphorischen Pronomen als Pro-Ergänzung.

\section{Zusammenfassung}

Ich habe eingangs die These aufgestellt, daß Konstruktionen mit ProW als effiziente Produktionsstrategien betrachtet werden können, obwohl die Beispiele zeigen, daß es sich dabei immer um Expansionen im Vergleich zum Input handelt, also um mehr Wortmaterial, was eigentlich auch mehr Verarbeitungskapazität beanspruchen müßte.

Aber was bei der Verarbeitungsleistung die entscheidende Rolle spielt, ist nach Hoffmann (1982) "nicht die Anzahl der zu speichernden Einheiten, sondern die Intensität der aufzuwendenden Prozesse".7 Diese Hypothese der Gedächtnisforschung läßt sich auch auf die Sprachproduktion übertragen.

Es scheint sich bei den Vorkommensfällen von ProW im Beispielmaterial um nicht-verarbeitungsintensive Produktionsstrategien zu handeln. Dafür sprechen folgende Beobachtungen:

ProW-Konstruktionen sind oft Standardstrukturen. Die Häufigkeit ihres Gebrauchs erhöht die leichte Abrufbarkeit aus dem Gedächtnis. Dazu kommt, daß Funktionswörter wie Pronomen im Dänischen (wie in vielen anderen Sprachen) zum Bestand der frequentesten Wörter überhaupt gehören.

Die syntaktischen Standardumgebungen der anaphorischen Proformen stimmen mit bevorzugten Konstruktionsweisen der spontanen gesprochenen Sprache überein (vor allem kürzere Einheiten, weniger Subordi-

7 Zitiert bei Salevsky (1986:42). 
nierung, häufige Anwendung derselben wenigen Konnektoren, besonders des nebenordnenden "und", bzw. gänzlicher Verzicht auf Konnektoren, parataktische Strukturierung).

Die anaphorischen Proformen beziehen sich auf Einheiten mit hohem Aktiviertheitsgrad im Gedächtnis, allein schon aufgrund der linearen Nähe (das Antezedens befindet sich innerhalb derselben Äußerungseinheit oder im Vorgängersatz).

ProW-Konstruktionen erfüllen im on-line-Prozeß oft mehrere Funktionen gleichzeitig (z.B. Korrektur und Zusammenfassung, Zusammenfassung und Verständnissicherung). Das erhöht ihre Effizienz.

ProW-Konstruktionen werden gleichermaßen von routinierten und weniger routinierten Dolmetschern verwendet, wenn auch z.T. in unterschiedlichen Funktionen (z.B. wird paraphrasierende Wiederaufnahme als Ersatz für weggefallene AS-Information, d.h. ProW mit der Funktion Kaschierung von Unsicherheit/Gedächtnisdefizit, überwiegend von den nicht-routinierten Dolmetschern benutzt).

Vor dem Hintergrund dieser Beobachtungen erscheint es mir gerechtfertigt, die Wiederaufnahme durch Proformen als Universalmittel zu charakterisieren, das beim SD aus dem Deutschen unentbehrlich ist.

\section{Literatur}

Bastian, Sabine (1991): Das Normenproblem in der französischen Sprache der Gegenwart unter besonderer Berücksichtigung des "français parlé". Ein Beitrag zur Determination von Lehrnormen für die Aus-und Weiterbildung von Sprachmittlern. Diss., Karl-Marx-Universität Leipzig.

Beaugrande, Robert A. de \& Dressler, Wolfgang U. (1981): Einführung in die Textlinguistik. Tübingen: Niemeyer.

Bußmann, Hadumod (1990): Lexikon der Sprachwissenschaft. Zweite, völlig neu bearbeitete Aufl. Stuttgart: Kröner.

Galberg Jacobsen, Henrik \& Skyum-Nielsen, Peder (1988): Erhvervsdansk. Grundbog. 2. überarb. Aufl. Kopenhagen.

Halliday, Michael A.K. \& Hasan, Ruqaiya (1976): Cohesion in English. London/New York: Longman.

Heinze, Helmut (1979): Gesprochenes und geschriebenes Deutsch. Vergleichende Untersuchung von Bundestagsreden und deren schriftlich aufgezeichneter Version. Düsseldorf: Schwann (= Sprache der Gegenwart 47).

Salevsky, Heidemarie (1986): Probleme des Simultandolmetschens. Eine Studie zur Handlungsspezifik. Berlin (= Linguistische Studien des Zentralinstituts für Sprachwissenschaft der Akademie der Wissenschaften der DDR: A/154). 
